\title{
Upgrade of the ATLAS Tile Calorimeter High Voltage System
}

\author{
A. Gomes, ${ }^{a, b, 1}$ J. S. Augusto, ${ }^{a, c}$ F. Cuim, ${ }^{a}$ G. Evans, ${ }^{a, b}$ R. Fernandez, ${ }^{a}$ L. Gurriana, ${ }^{a}$ R. \\ Marques, ${ }^{a, b}$ F. Martins, ${ }^{a}$ and C. Pereira ${ }^{a, b}$ on behalf of the ATLAS Tile Calorimeter group \\ ${ }^{a}$ LIP, Lisboa, Portugal \\ ${ }^{b}$ Faculdade de Ciências da Universidade de Lisboa, Lisboa, Portugal \\ ${ }^{c}$ INESC-ID, Lisboa, Portugal \\ E-mail: agomes@lip.pt
}

\begin{abstract}
The High Voltage system of TileCal, the ATLAS central hadron calorimeter, is being upgraded for the High-Luminosity LHC, in the so called Phase II Upgrade. In the new configuration for the upgrade, the high voltage regulation boards are not located inside the detector anymore, they are deployed far from the radiation caused by the collisions, in a room where there is permanent access for maintenance. This option requires a large number of $100 \mathrm{~m}$ long high voltage cables but removes the requirement of radiation hardened boards. HVremote regulation boards and the respective high voltage supplies boards have been developed and tested, as well as a crate to house the boards. Preliminary results of the HVremote boards performance are presented.
\end{abstract}

KeYwords: Calorimeters; voltage distribution

${ }^{1}$ Corresponding author.

Copyright 2021 CERN for the benefit of the ATLAS Collaboration. CC-BY-4.0 license. 


\section{Contents}

1 Introduction 1

2 High Voltage system design 1

3 Control and monitoring 3

4 HVremote prototypes and performance 3

5 Conclusions and next steps 5

\section{Introduction}

The Tile calorimeter (TileCal) is the central hadron calorimeter of ATLAS [1]. It is made of steel and plastic scintillator tiles oriented perpendicularly to the beam. Wavelength shifting fibres collect the light on both sides of the scintillators and transport it to the photomultiplier tubes (PMTs), two PMTs per calorimeter cell. The calorimeter is divided into 4 partitions, each one composed of 64 modules. The PMTs and Front End electronics are mounted in $3 \mathrm{~m}$ long drawers at the outer radius of the modules. For operation at the High-Luminosity Large Hadron Collider (HL-LHC), the ATLAS Phase II upgrade is in progress. The HL-LHC is planned to deliver more than ten times the integrated luminosity of the LHC Runs 1, 2 and 3 combined. The higher radiation levels due to the higher luminosity and the ageing of the TileCal high voltage system require the development of a new HV system [2].

\section{High Voltage system design}

The High Voltage system of TileCal after the Phase II upgrade will consist of HVremote boards and HV supplies boards, located far from the detector in crates, connected to the detector by $100 \mathrm{~m}$ long cables [3]. Inside the detector there will be passive HVbus boards that are used to bring the HV to each of the PMTs located in mini-drawers inside the girders of the modules. The HVremote boards are the boards that regulate the HV for each individual PMT of TileCal using the regulation loop shown in Figure 1. This regulation loop is an adapted version of the one that is used in the HV system that operates currently in TileCal [4], with minor changes since the operation in the absence of radiation allowed the simplification of the regulation loops with the removal of some transistors. It is required that the high voltage fluctuations in the PMTs are lower than $0.5 \mathrm{~V}$ (rms). The number of HVremote boards needed for the TileCal operation is 256. Relative to the current TileCal HV system, the main functional improvement is the addition of on/off control for each group of 4 channels complemented by a jumper for each individual channel. 


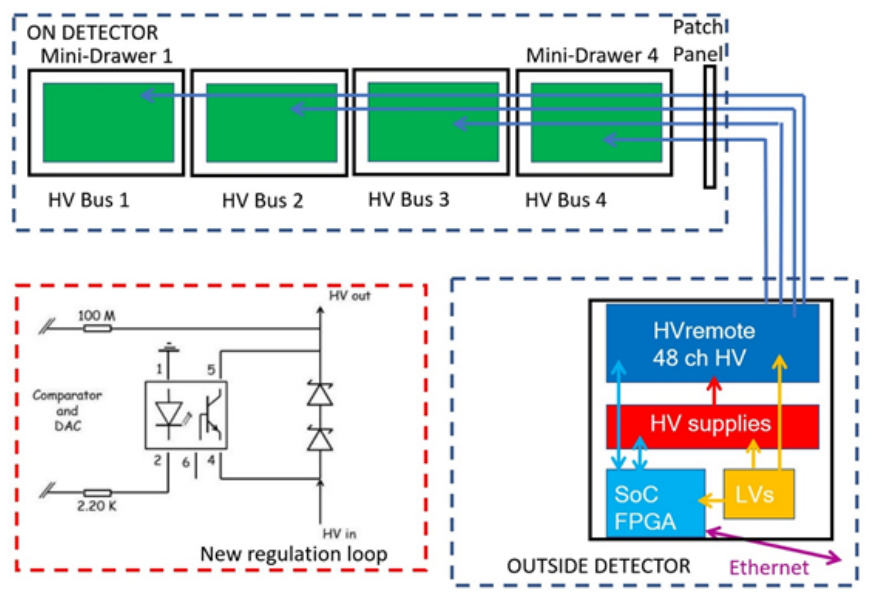

Figure 1. High voltage for Phase II upgrade. The regulation system is remote, located far from the detector, and a large number of $100 \mathrm{~m}$ long cables bring the $\mathrm{HV}$ to the detector modules. Inside the detector, the HV is distributed to 4 (3) mini-drawers in the Long Barrel (Extended Barrel) modules. The main part of the new regulation loop is very similar to the current one [3].

There is one input HV (-830 or $-950 \mathrm{~V}$ ) for each 24 channels and the voltage of each channel can be regulated down individually in a range of the order of $360 \mathrm{~V}$ as in the current TileCal design [3, 4]. The primary HV is provided by Hamamatsu C12446-12 modules located in the HV supplies boards, and two primary HV inputs are used to provide HV for 48 channels in the case of the Barrel modules or for 32 channels in the case of the Extended Barrel ones, as can be seen in Figure 2 (right) where the first prototype of the HV supplies board is shown. A crate prototype to house the HV supplies and HVremote boards was already produced and is seen in Figure 2 (left). The crates house the HVremote boards in the back and the HV supply boards in front, plus a control board and low voltage power supplies $(+24 \mathrm{~V},+12 \mathrm{~V},-12 \mathrm{~V}$ and $+3.3 \mathrm{~V})$.

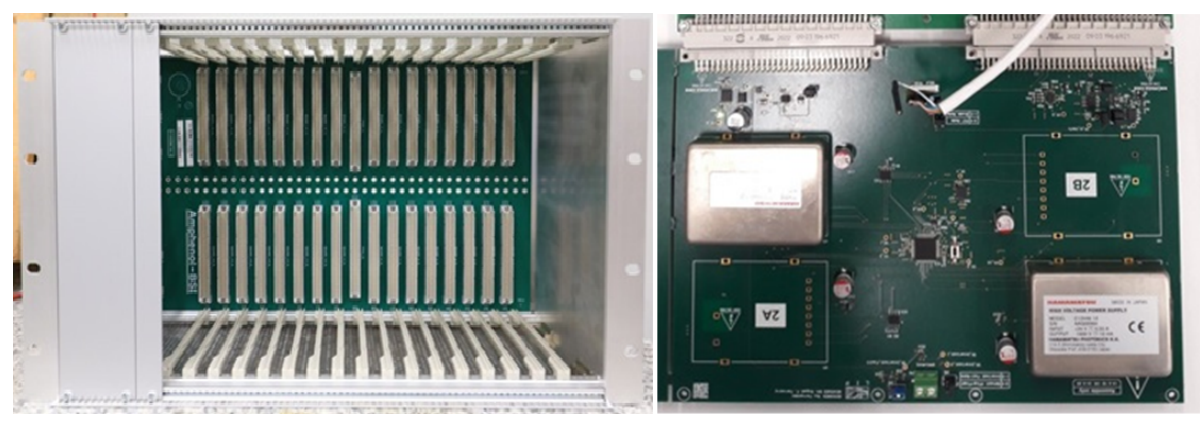

Figure 2. (left) Prototype of the crate for the boards. It has a midplane and the HVremote boards go in the back. (right) Prototype of HV supply board. It has two DC-DC Hamamatsu C12446-12 modules, each one able to supply $10 \mathrm{~mA}$ up to $-1000 \mathrm{~V}$.

The only components of the HV distribution system that are to be used inside the detector are the HVbus boards. They are fully passive, with 4 layers to have the HV tracks protected in the inner layers. The last prototype was produced for the TileCal testbeams of 2021 and 3 boards are shown in Figure 3. 


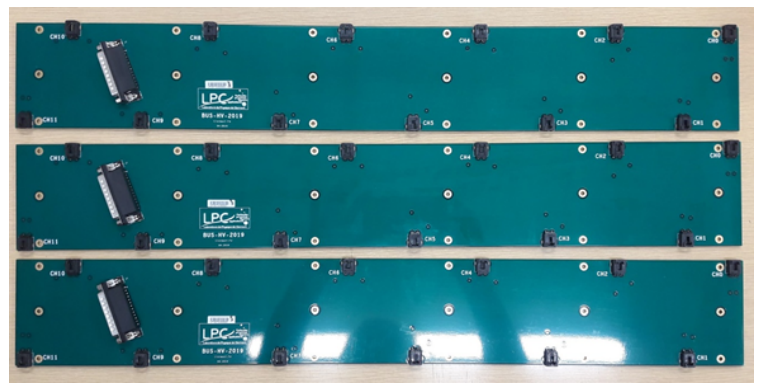

Figure 3. Set of 3 HVbus prototypes produced this year following the design from LPC Clermont Ferrand.

\section{Control and monitoring}

The control and monitoring system, that in the current TileCal version is done with HVmicro boards [4] that communicate with the detector control system PCs via CANbus, was completely redone. The communication and control is done via Serial Peripheral Interface (SPI) bus through an Ethernet interface. Most of the tests and development of the control and monitoring of the HVremote and HV supply boards was done using Raspberry Pi platform, with the solutions being later moved to a System on Chip solution (Zybo Z7 Zynq) that allows the simultaneous usage of two SPI buses, one for the HVremote boards and the other one for the HV supply boards [5].

\section{HVremote prototypes and performance}

Several prototypes of the HVremote board with different configurations were already produced [3]. The last version has 48 channels and is shown in Figure 4 where it is being tested with an adapter board and a HV supplies board. The first version with 48 channels had the outputs of 4 multiplexers connected in the same point and we experienced the damage of some multiplexers in events in which one of the +/- $12 \mathrm{~V}$ went off while the symmetric voltage was kept on. We observed that in such circumstances the multiplexers would get very hot and in some cases could get damaged. A very useful tool for the identification of the problem was a thermal camera that helped to monitor the temperature of the components of the boards in operation as seen in Figure 5. In normal operation the hottest components are the OpAmps used in the regulation loops shown as two brighter lines along the central region of the HVremote board, at the right side of Figure 5. In the last prototype an additional multiplexer was used to decouple the output lines of the other multiplexers of the board and until now we have no further evidence of damage in the multiplexers in the new boards.

The last version of the HVremote board was tested in the laboratory in the setup shown in Figure 4 and most of the channels have shown to be very stable as it is seen in Figure 6 where channel 13 is used as example. In the laboratory results, only one channel suffers from low amplitude fluctuation up to the order of $1 \mathrm{~V}$, channel 12 also shown in Figure 6. This HVremote board was calibrated both for control (voltage vs DAC value, $V=$ calib. factor $\times D A C+o f f s e t$ ) and monitoring (voltage vs $\mathrm{ADC}$ value, $V=$ calib. factor $\times A D C+$ offset). Calibration results for the first 10 channels are shown in table 1. This board was used for the first time in a testbeam in September 2021 and very preliminary results show stable voltages in 45 channels that were well connected to the HVbus boards, with rms values in the range $0.2-0.3 \mathrm{~V}$ in a five hour run. 


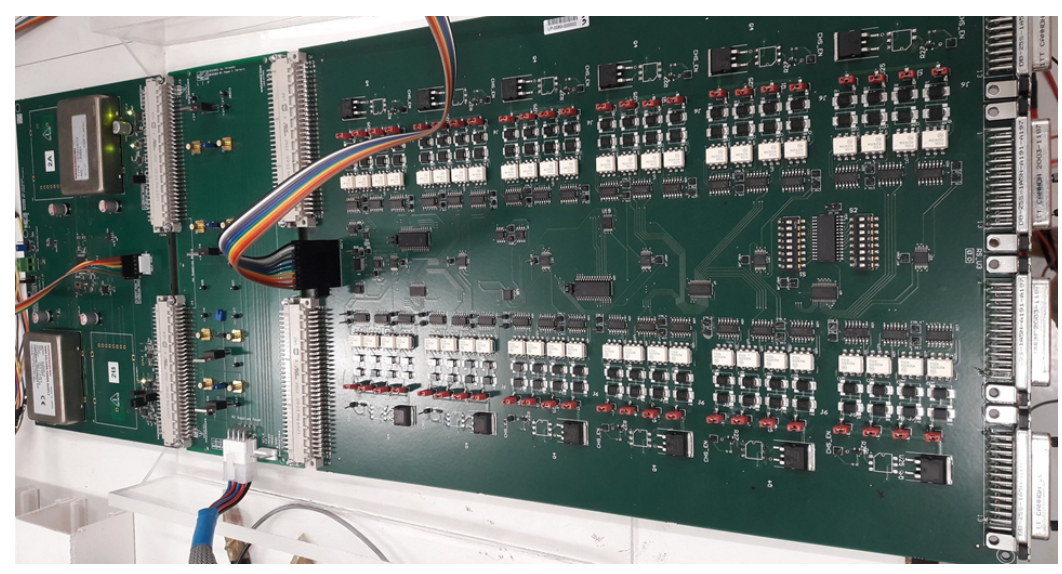

Figure 4. Test setup with HV supplies board at left, adapter board at center and HVremote board at right.

Table 1. HVremote board prototype readings and settings calibration for 10 channels of the HVremote board prototype. ADC and DAC values in range from 0 to 4095.

\begin{tabular}{|c|c|c|c|c|}
\hline & \multicolumn{2}{|c|}{ V as function of ADC value } & \multicolumn{2}{c|}{ V as function of DAC value } \\
\hline channel & calibration factor & offset & calibration factor & offset \\
\hline Ch 1 & 0.2511 & -3.8550 & 0.2407 & 0.6491 \\
Ch 2 & 0.2511 & -4.1186 & 0.2421 & 0.5651 \\
Ch 3 & 0.2512 & -3.7124 & 0.2426 & 0.5581 \\
Ch 4 & 0.2496 & -3.7878 & 0.2414 & 0.7552 \\
Ch 5 & 0.2502 & -3.8007 & 0.2415 & 0.1809 \\
Ch 6 & 0.2518 & -3.0491 & 0.2420 & 0.1841 \\
Ch 7 & 0.2508 & -3.7339 & 0.2422 & 0.2635 \\
Ch 8 & 0.2504 & -3.7130 & 0.2416 & 0.2891 \\
Ch 9 & 0.2525 & -3.8069 & 0.2426 & 0.5787 \\
Ch 10 & 0.2508 & -3.5158 & 0.2422 & 0.5083 \\
\hline
\end{tabular}

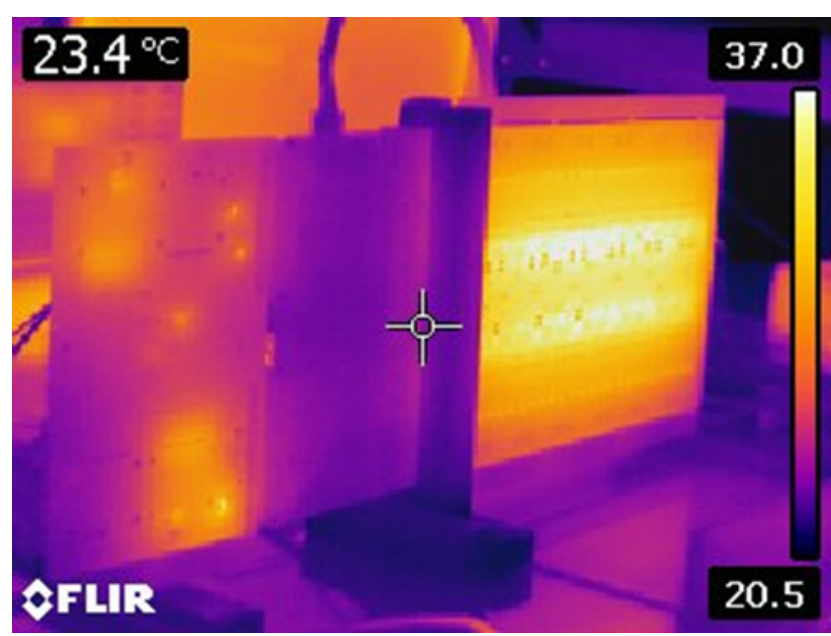

Figure 5. Temperature distributions of HV supplies at the left side of the picture and HVremote boards at the right side of the picture. 


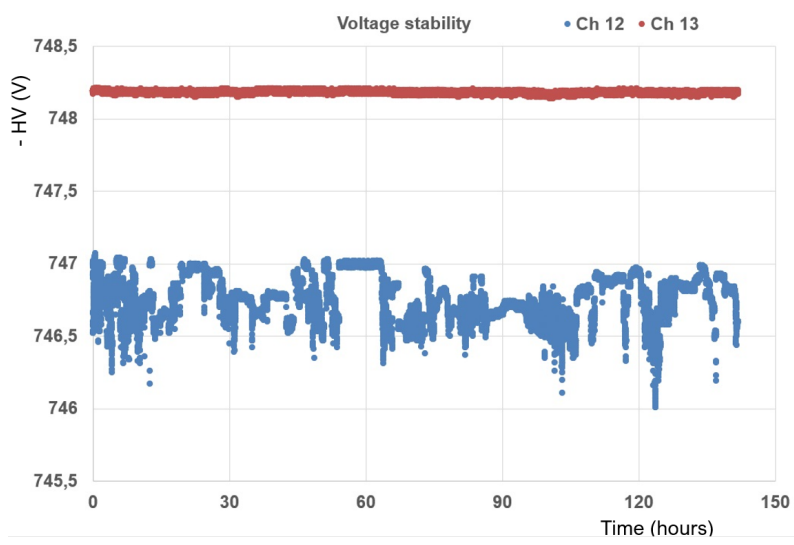

Figure 6. Voltage readings from channel 12 and channel 13 of the 48 channel prototype of HVremote board (readings every $10 \mathrm{sec}$ using a voltmeter). Channel 13 is very stable and 12 is unstable but within $+/-0.5 \mathrm{~V}$. This prototype board has only one slightly unstable channel (ch. 12) and one bad channel to be repaired.

\section{Conclusions and next steps}

Prototypes of almost all the elements needed for the upgrade of the HV system of TileCal (HVremote, HV supplies, HVbus, crate, cables) were already produced. Preliminary results show that all of them are functional and produce the HVs needed for the PMTs and the HVremote boards regulate the voltages to the PMTs within the specifications. A new set of prototypes is being designed for production in the next few months, and it is expected to test a new HVremote board in the testbeam still in 2021 and the other boards and cables after the testbeam and in early 2022 in a full slice of the HV system.

\section{Acknowledgments}

The authors would like to thank P. Assis, M. Ferreira and J.C. Nogueira from LIP eCRLab for all the general support and in particular in the HV supplies board development. We also thank R. Bonnefoy from LPC Clermont-Ferrand for the support in the HVbus. This work was supported in part by the Portuguese Fundação para a Ciência e Tecnologia, CERN/FIS-PAR/0033/2019.

\section{References}

[1] ATLAS Collaboration, The ATLAS experiment at the CERN Large Hadron Collider, JINST 3 (2008) S08003, https://cds.cern.ch/record/1129811/files/jinst8_08_s08003.pdf

[2] ATLAS Collaboration, Technical Design Report for the Phase-II Upgrade of the ATLAS Tile Calorimeter, CERN-LHCC-2017-019 (2017), https://cds.cern.ch/record/2285583

[3] A. Gomes et al., Upgrade of the ATLAS Tile Calorimeter High Voltage System, PoS 370 (2020) 062, https://pos.sissa.it/370/062/

[4] R. Chadelas et al., High voltage distributor system for the Tile hadron calorimeter of the ATLAS detector, ATLAS-TILECAL-2000-003 (2000), https://cds.cern.ch/record/436230

[5] F. Martins et al., Control system for ATLAS TileCal HVRemote boards, in Proceedings, of ICALEPCS 2017 (2018), https://doi.org/10.18429/JACoW-ICALEPCS2017-THPHA069 